\title{
Der „Fall“ Eggebrecht
}

Verzeichnis der Veröffentlichungen in chronologischer Folge 2009-2013 zusammengestellt von Matthias Pasdzierny, Johann Friedrich Wendorf und Boris von Haken

alle URLs abgerufen am 16.7.2013

\section{9}

Boris von Haken: Spalier am Mördergraben, in: DIE ZEIT, 17.12.2009

[Online-Version inkl. Leser-Kommentare unter: http://www.zeit.de/2009/52/EggebrechtKriegsverbrechen]

Volker Hagedorn: Unheimliches Abendland, in: DIE ZEIT, 17.12.2009

Arno Lücker: Wer war Hans Heinrich Eggebrecht? Oder: Das Zwei-Welten-Modell mal ganz anders..., in: Bad Blog of Musick, 17.12.2009, http://blogs.nmz.de/badblog/2009/12/17/ wer-war-hans-heinrich-eggebrecht/

Alexander Dick: Die zwei Leben - War der Musikwissenschaftler Hans Heinrich Eggebrecht 1941 an Erschießungen beteiligt?, in: Badische Zeitung, 18.12.2009

Lorenz Jäger: Der Feldgendarm, in: $F A Z, 18.12 .2009$

epd: Musikhistoriker Eggebrecht an NS-Verbrechen beteiligt, in: Die Welt, 18.12.2009

Berthold Schossig: „Der Fall Eggebrecht ist etwas Besonderes“. Musikwissenschaftler über die Enthüllungen im Fall Hans Heinrich Eggebrecht. Boris von Haken im Gespräch mit Rainer Berthold Schossig, Deutschlandradio/Deutschlandfunk, Kultur Heute, 18.12.2009 http://www.dradio.de/dlf/sendungen/kulturheute/1091605/drucken/

Jens Malte Fischer: Unterstellung ohne Belege. NS-Vorwürfe gegen Musikwissenschaftler H. H. Eggebrecht, in: Süddeutsche Zeitung, 19.12.2009.

Kai Luehrs-Kaiser: Unbehelligte Karriere. Der Musikwissenschaftler Hans Heinrich Eggebrecht war 1941 an der Erschießung von Juden beteiligt, in: Die Welt, 21.12.2009

Ulrike Timm: „Rechtsstaatliche Prinzipien werden mit Füßen getreten“. Kulturwissenschaftler gegen Vorverurteilung von potenziellem Nazi-Verbrecher. Jens Malte Fischer im Gespräch mit Ulrike Timm. Deutschlandradio/Deutschlandradio Kultur, Radiofeuilleton Thema, 22.12.2009, http://www.dradio.de/dkultur/sendungen/thema/1092668/ drucken

Kai Luehrs-Kaiser: Wie Eggebrecht seine Nazi-Zeit totschwieg, in: Die Welt, 23.12.2009

Friedrich Geiger: Im langen Schatten deutscher Musik. Der auf Deutschland beschränkte Blick vieler Musikwissenschaftler ist das eigentliche Problem - jenseits der individuellen Verfehlungen, in: FAZ, 23.12.2009

Richard Klein: Suche nach einer Sprache in der falschen Welt. Über das Verhältnis von allgemeiner deutscher Ideologie und spezifischem Nationalsozialismus, in: FAZ, 23.12.2009

Lorenz Jäger: Ein Gespräch mit Götz Aly. Die Täter waren nicht primitiv, in: $F A Z$, 23.12.2009

Alexander Dick: „Perfekter Rufmord“ - Benjamin Eggebrecht zu den Vorwürfen an seinem Vater, in: Badische Zeitung, 30.12.2009 
Stephan Hoffmann: Der Musikwissenschaftler Hans H. Eggebrecht soll an NS-Verbrechen beteiligt gewesen sein, in: Südkurier, 30.12.2009

Stephan Hoffmann: Der späte Verlust einer Vaterfigur. Kriegsverbrechen: Der Musikwissenschaftler Hans Heinrich Eggebrecht soll an Juden-Erschießungen beteiligt gewesen sein, in: Kölner Stadt-Anzeiger, 31.12.2009

Thomas Meyer: Hans Heinrich Eggebrecht, in: Musik-Texte 123 (2009), S. 96

\section{0}

hu: Fall Eggebrecht erhitzt die Gemüter - Ein Musikwissenschaftler als Massenmörder? Warnung vor Vorverurteilung, in: Badische Zeitung, 3.1.2010

Boris von Haken: Musicology and mass execution, in: signandsight.com, 5.1.2010, http:// www.signandsight.com/features/1977.html [engl. Übersetzung des ZEIT-Artikels vom 19.12.2009]

Alexander Dick/Johannes Adam: „Die Beweislage ist nicht lückenlo““ - BZ-Interview: Boris von Haken über eine mögliche Beteiligung des Musikologen Hans Heinrich Eggebrecht an Kriegsverbrechen, in: Badische Zeitung 9.1.2010

Gisela Spieß: Fall Eggebrecht. Wo bleibt denn da die Moral? [Leserbrief], in: Badische Zeitung, 9.1.2010

Frieder Reininghaus: Tabuthema NS in der Musik. Die Entnazifizierung in der Musikwissenschaft, in: Deutschlandradio/Deutschlandfunk, Kultur Heute 10.1.2010, http:// www.dradio.de/dlf/sendungen/kulturheute/1102459/drucken

Peter Wivel: Historiker anklages for mord på jøder. Fortiden indhenter en af Tysklands mest anerkendte musikhistorikere, in: Politiken, 10.1.2010

Lorenz Jäger: Eggebrechts Akten. Geöffnet für die Forschung, in: FAZ, 13.1.2010

Boris von Haken: Erdrückende Quellenlage, in: DIE ZEIT, 14.1.2010

Das schlug wie eine Bombe ein. Boris von Haken: „Spalier am Mördergraben“ und Volker Hagedorn: „Unheimliches Abendland“, in: DIE ZEIT, 14.01.2010 [Leserbriefe von Christoph Eggebrecht, Wilfried Gruhn, Reinhold Bertscheit, Sascha Wegner, Ulrich Mahlert]

Gertraude Franz, geb. Eggebrecht: Haben wir nicht aus der Vergangenheit gelernt? [Leserbrief], in: Badische Zeitung, 21.1.2010

Jürgen Kaube: Neues zum Fall Hans Heinrich Eggebrecht. Der Musikwissenschaftler als Massenmörder: Beweis aus den Quellen oder Skandalphilologie, die nur Belastungsmomente heranzieht und Gewissheit fingiert? in: FAZ, 17.3.2010

Christiane Tewinkel: Strategische Rehabilitierung als politische Kunstform. Der kann doch kein Nazi gewesen sein: Adorno und der Musikwissenschaftler Joseph Müller-Blattau, in: $F A Z, 24.3 .2010$

Alexander Dick: Kommentar: Ein Musikwissenschaftler stochert im Trüben. Zweifel an den Vorwürfen gegenüber Hans Heinrich Eggebrecht, in: Badische Zeitung, 1.4.2010

Wolfram Wette: Das Phänomen. Nach 1945 verschwinden die Täter, in: Badische Zeitung, 15.4.2010

Irmfried Brendel: Eine Krähe hackt der anderen kein Auge aus [Leserbrief], in: Badische Zeitung, 21.4.2010

Hans-Joachim Michel: Eggebrecht war in der falschen Kompanie [Leserbrief], in: Badische Zeitung, 21.4.2010 
Frieder Reininghaus: Zur neueren deutschen Bewegungslehre: Die Entnazifizierung der Musikforschung und -publizistik, in: Neue Zeitschrift für Musik 171 (2010) 2, S. 52-54

Boris von Haken: Holocaust und Musikwissenschaft: Zur Biographie von Hans Heinrich Eggebrecht, in: Archiv für Musikwissenschaft 67 (2010) 2, S. 146-163

Albrecht Riethmüller: Editorial, in: Archiv für Musikwissenschaft 67 (2010) 2, S. 1-2

Reinhard Oehlschlägel: Ohne hieb- und stichfeste Beweise: zur Causa Eggebrecht, in: $M u$ sik-Texte 125 (2010), S. 92

Albrecht von Massow: Gehversuche musikwissenschaftlicher Vergangenheitsbewältigung, Online-Publikation, Weimar 2010, http://www.albrecht-von-massow.de

Friedrich Geiger: Quellenkritische Anmerkungen zum „Fall Eggebrecht“, Online-Publikation, Hamburg 2010, http://www.uni-hamburg.de/Musikwissenschaft/buch_/geiger_ eggebrecht.pdf

Claudia Maurer Zenck: Eggebrechts Militärzeit auf der Krim, Online-Publikation, Hamburg 2010, http://www.uni-hamburg.de/Musikwissenschaft/buch_/zenck_eggebrecht. pdf

Richard Klein: Der Fall Eggebrecht und die deutsche Musikwissenschaft, in: Merkur. Deutsche Zeitschrift für europäisches Denken 64 (2010) 4, S. 325-331

Alexander Dick: „Er hat viele junge Wissenschaftler unterstützt“ - BZ-Interview mit Professor Günter Schnitzler zur Podiumsdiskussion „Der ,Fall' Hans Heinrich Eggebrecht?“ am Mittwoch, in: Badische Zeitung, 8.6.2010

Johannes Adam: „Er muss sehr viel gewusst haben“. Der „Fall“ Hans Heinrich Eggebrecht: Eine Podiumsdiskussion in der Freiburger Universität galt dem umstrittenen Musikologen, in: Badische Zeitung, 11.6.2010

Diethard Köster: Was wirft man ihm denn genau vor? $\mathrm{Zu}$ : „Er muss sehr viel gewusst haben“, Beitrag von Johannes Adam (Kultur, 11. Juni) [Leserbrief], in: Badische Zeitung, 12.6.2010

Albin Eser: Warum sollte man nicht von einem moralischen Versagen sprechen dürfen? Zu: „Er muss sehr viel gewusst haben“, Beitrag von Johannes Adam (Kultur, 11. Juni). [Leserbrief], in: Badische Zeitung, 19.6.2010

Gertraude Franz, geb. Eggebrecht: Mein Bruder war sensibel genug, um die Schuld unserer Generation zu spüren. [Leserbrief] $\mathrm{Zu}$ : „Er muss sehr viel gewusst haben“, Beitrag von Johannes Adam (Kultur, 11. Juni), in: Badische Zeitung, 2.7.2010

\section{1}

Norman Lebrecht: Rezension von: Jens Malte Fischer: Gustav Mahler [New Haven 2011], in: New Statesman, 12.5.2011

Arnold Whittall: Rezension von: Hans Heinrich Eggebrecht: Understanding Music: The Nature and Limits of Musical Cognition, in: Music and Letters 92 (2011) 4, S. 677

Melanie Unseld: Musikwissenschaft als Kulturwissenschaft, Oldenburg 2011 (Oldenburger Universitätsreden 195)

\section{2}

Rafael Rennicke: Musikwissenschaft und NS. Klangarbeit, in: FAZ, 25.1.2012

Anne C. Shreffler: The Case of Hans Heinrich Eggebrecht: The Moral Dilemma of a Tainted

Past, in: German Studies Review 35 (2012) 2, S. 290-298 
Christopher R. Browning: An American Historian's Perspective, in: German Studies Review 35 (2012) 2, S. 310-318

Boris von Haken: How Do We Know What We Know about Hans Heinrich Eggebrecht?, in: German Studies Review 35 (2012) 2, S. 299-309

Gottfried Schramm/Günter Schnitzer (Hrsg.): Ein umstrittener Lebensweg. Muß der Freiburger Musikwissenschaftler Hans Heinrich Eggebrecht neu beurteilt werden? Freiburger Universitätsblätter 51 (2012) 195

Gottfried Schramm: Stichworte zum Heft, in: Freiburger Universitätsblätter 51 (2012) 195 , S. 7-8

Christian Berger: Hans Heinrich Eggebrecht, in: Freiburger Universitätsblätter 51 (2012) 195, S. 9-12

Albrecht von Massow: Gehversuche musikwissenschaftlicher Vergangenheitsbewältigung, in: Freiburger Universitätsblätter 51 (2012) 195, S. 13-50 [für den Druck bearb. Fassung der Online-Publikation von 2010]

Christopher R. Browning: Musikwissenschaft und Biographie: der Fall Hans Heinrich Eggebrecht aus der Perspektive eines amerikanischen Historikers, in: Freiburger Universitätsblätter 51 (2012) 195, S. 51-59 [dt. Fassung des Artikels aus der German Studies Review]

Christoph Wolff: Beredtes Schweigen und ein Klavier als ,smoking gun', in: Freiburger Universitätsblätter 51 (2012) 195, S. 61-65

Günter Schnitzler/Gottfried Schramm: Der Hochschullehrer Eggebrecht. Ein Gespräch [mit Christian Berger und Elmar Budde], in: Freiburger Universitätsblätter 51 (2012) 195, S. 67-72

Matteo Nanni: Erinnerungen an Gespräche mit Hans Heinrich Eggebrecht, in: Freiburger Universitätsblätter 51 (2012) 195, S. 73-74

Hans Peter Herrmann: Vom Umgang mit der NS-Vergangenheit: der „Fall Eggebrecht“, die Universität Freiburg und die Etappen deutscher Erinnerungspolitik 1957-2005, in: Freiburger Universitätsblätter 51 (2012) 195, S. 75-113

Bettina Schulte: Vergangenheit, die nicht vergeht. Zu ihrem 50-Jährigen beschäftigen sich die Freiburger Universitätsblätter mit Hans H. Eggebrecht, in: Badische Zeitung, 28.4.2012

Dörte Schmidt: Musikwissenschaft und Vergangenheitspolitik, in: Geschichte der Germanistik. Mitteilungen 41/42 (2012), S. 102-108

Helmut Mauró: Charakterlich geprüft und doch ein Hetzer. Kampfbündler Friedrich Blume: Es gibt jetzt handfeste Beweise für die Nazi-Vergangenheit des einflussreichsten deutschen Musikwissenschaftlers, in: Süddeutsche Zeitung, 9.5.2012

Albrecht Dümling: Musikwissenschaftler als ideologische Hetzer. Zu den „Fällen“ Hans Heinrich Eggebrecht und Friedrich Blume, in: nmz - neue musikzeitung, 7.9.2012

dg: Der Fall Eggebrecht: Analyse einer Hetzjagd, in: Junge Freiheit, 16.11.2012

\section{3}

Peter Sühring: Ein umstrittener Lebensweg. Muß der Freiburger Musikwissenschaftler Hans Heinrich Eggebrecht neu beurteilt werden? [Rezension Freiburger Universitätsblätter 51 (2012)], info-netz-musik 14.1.2013, http://info-netz-musik.bplaced. net/?p=8517\#sthash.v3fSUDMf.dpuf

„Der Fall“ Eggebrecht: Noch einmal, Musik \& Ästhetik 67 (Juli 2013) 
Richard Klein: Einleitung, in: Musik \& Ästhetik 67 (2013), S. 5-8

Ulrike Jureit: In dubio contra reum? Über den Wunsch nach historischer Eindeutigkeit, in: Musik \& Ästhetik 67 (2013), S. 9-21

Richard Klein: Klavierspielen in Simferopol. Der „Fall Eggebrecht“ und die deutsche Musikwissenschaft, in: Musik \& Ästhetik 67 (2013), S. 22-37

Friedrich Geiger: Quellenkritische Anmerkungen zum »Fall Eggebrecht«, in: Musik \& Ästhetik 67 (2013), S. 38-55 [überarbeitete Fassung der Online-Publikation von 2010]

Claudia Maurer Zenck: Der "Fall Eggebrecht« aus biographischer Sicht. Eine Kritik, in: Musik \& Ästhetik 67 (2013), S. 56-76 [überarbeitete Fassung der Online-Publikation von 2010]

Simon Obert/Matthias Schmidt: Die Leerstelle der Historiographie. Zur Praxis der Geschichtsreflexion im „Fall Eggebrecht“, in: Musik \& Ästhetik 67 (2013), S. 77-96

Rainer Bayreuther: NS-Musikwissenschaft ex negativo. Orgel- und Schützbewegung bei Hans Heinrich Eggebrecht, in: Musik \& Ästhetik 67 (2013), S. 97-114

Alexander Dick: Die Scherben aufkehren. „Der Fall Eggebrecht“ im Juli-Heft „Musik \& Ästhetik“, in: Badische Zeitung, 9.7.2013

Volker Hagedorn: Im Herzen das größte Grauen, in: DIE ZEIT, 11.7. 2013

Christian Wildhagen: „Beethoven“. Eggebrecht, noch einmal, in: FAZ, 31.7.2013

Lotte Thaler: Der prekäre Fall Eggebrecht [Leserbrief], in: DIE ZEIT, 25.7.2013 